\title{
Density of states of one-dimensional Pauli ionic conductor
}

\author{
I.V.Stasyuk, I.R.Dulepa \\ Institute for Condensed Matter Physics of the National Academy of Sciences of Ukraine, \\ 1 Svientsitskii Str., 79011 Lviv, Ukraine
}

Received May 7, 2007

\begin{abstract}
Microscopic one-dimensional noninteracting model for the description of the energy spectrum of the ion subsystem in ionic conductor is considered. The processes of ionic hoppings are described in terms of Pauli operators. Time-dependent correlation functions $\left\langle b(t) b^{+}(0)\right\rangle_{j}$ in Pauli operators are obtained using the exact numerical procedure known for time-dependent spin correlation functions. The frequency dependence of autocorrelation function $J_{b b^{+}}(\omega)$ is calculated and analysed at the wide range of temperatures. The frequency and temperature dependences of the one-particle density of states are investigated.
\end{abstract}

Key words: Pauli statistics, correlation functions, particle density of states, ionic conductor

PACS: $75.10 . P q, 66.30 . D n, 66.10 . E d$

\section{Introduction}

An ion transfer in solids has become an active area of research nowadays. Different theoretical models have been developed to perform the necessary investigations in this direction. One of the most important aspects in this field is the problem of determining the energy spectrum, e. g. the particle density of states, and the ionic conductivity. The latter is connected with the process of site-to-site ionic hopping and strongly depends on temperature. It is important to mention here that the activation energy of conductivity is determined not only by the height of potential barrier which the ion must overcome changing its position but is also effected by the nearest-neighbor configuration. An interaction energy should be taken into account in theoretical investigations of the ionic hopping in solids.

Among several basic models of ionic hopping conduction, the lattice gas model [1-5] is the mostly used. This model has been introduced by G. D. Mahan three decades ago to calculate the ionic conductivity in superionic conductors. The conductivity value obtained was in agreement with the found experimental data [1]. At present the lattice gas model is applied, besides ionic conductors (see, for example, [6]), to numerous physical processes such as intercalation [7], adsorption [8] and others.

Perturbation expansion in powers of hopping parameter $[2,5]$ was used to calculate the correlation functions for the lattice gas model. Mostly, only the first order perturbation corrections were taken into account, i.e. the term which should describe the ionic hopping is neglected in the model Hamiltonian at statistical averaging. The second order perturbation expansion was taken into account in [5]. However, the nearest-neighbor interaction between ions was not considered. Taking into account the known exact solutions for Ising model, an explicit expression for the time dependent correlation function was obtained within the framework of lattice gas model [1] using current density operators to determine the ionic conductivity. However, the approach was applicable only in a narrow interval of the ion concentrations. Expansion in terms of the hopping parameter was also used in [9] in describing the conductivity of superprotonic conductor; the effect of phonon assisted hopping on the activation energy of protonic conductivity was taken into account. Summarizing, one can conclude that the problem of developing the model approaches that explicitely take into account the ionic hopping in the zero-order Hamiltonian still remains unresolved. 
Statistics of particles, which are described by the lattice gas model, as well as its consequences turn out to be another interesting question. In a series of papers devoted to ionic conductivity, starting with [1], the particles were described by Pauli creation and annihilation operators. This corresponds to an ordinary restriction on the ion occupation number at a certain lattice site and reflects the Bose origin of particles (the approach that corresponds to the picture of hardcore bosons [10]). Besides, the spinless fermion lattice model, where particles obey the Fermi statistics, is also used in describing the objects of the ionic conductor type [11]. The comparison of the mentioned approaches has not been made so far. In particular, it touches upon the possible difference in the energy spectrum structure and thermodynamics of the model.

In the present paper we consider the one-dimensional model of the ion hopping between nearest sites. For simplicity the nearest-neighbor interactions between ions are neglected. Our aim is to calculate the correlation functions and analyse the frequency dependences of the one-particle densities of states in the case when ions on the lattice are described using the Pauli statistics. The Pauli correlation functions are obtained using the exact numerical method based on the pseudospin representation and the fermionization procedure ${ }^{1}$.

In section 2 we consider the relation between pseudospin and Pauli operators; the model is discussed and the Jordan-Wigner transformation is applied. The time-dependent autocorrelation functions built on Pauli operators are obtained in section 3. This makes it possible to obtain frequency dependent densities of states at a given site, temperature and local energy of particles. The frequency and the temperature dependence of the density of states are analysed in section 4 . The comparison is made with the results that can be obtained within the fermion lattice model.

\section{Model}

The ion transfer from the given site to nearest-neighbor sites of a lattice in one dimension is described by the Hamiltonian

$$
H=\sum_{i=1}^{N}\left(\varepsilon_{0}-\mu\right) b_{i}^{+} b_{i}+t_{0} \sum_{i=1}^{N}\left(b_{i}^{+} b_{i+1}+b_{i+1}^{+} b_{i}\right)
$$

where $\varepsilon_{0}$ is the ion energy at the site, $\mu$ is chemical potential. We consider the chain with $N$ sites, $t_{0}$ is the transfer parameter.

The ions on the sites are described by the Pauli creation and annihilation $b_{i}^{+}$and $b_{i}$ operators which are neither fermion nor boson operators, but correspond exactly to $S=1 / 2$ spin operators. The ion presence or absence at the $i$-th site is equivalent to the mathematical problem with spin up or down. The commutation relations for the Pauli operators are as follows:

$$
\left\{b_{i}^{+}, b_{i}\right\}=1,\left[b_{i}^{+}, b_{j}^{+}\right]=\left[b_{i}, b_{j}\right]=\left[b_{i}^{+}, b_{j}\right]=0, \quad\left(b_{i}\right)^{2}=\left(b_{i}^{+}\right)^{2}=0 .
$$

We perform the transformation from these operators to spinless Fermi operators on the same lattice by means of the Jordan-Wigner transformation:

$$
b_{i}=S_{i}^{-}=a_{i}(-1)^{\sum_{j \leqslant i-1} n_{j}}, \quad b_{i}^{+}=S_{i}^{+}=a_{i}^{+}(-1)^{\sum_{j \leqslant i-1} n_{j}}
$$

and $\left[b_{i}^{+}, b_{i}\right]=2 S_{i}^{z}, S_{i}^{z}=b_{i}^{+} b_{i}-1 / 2=n_{i}-1 / 2 . a_{i}^{+}, a_{i}$ are Fermi operators. The sign factor can be written as follows:

$$
(-1)^{\sum_{j \leqslant i-1} n_{j}}=\prod_{j=1}^{i-1}\left(1-2 a_{j}^{+} a_{j}\right)=\prod_{j=1}^{i-1}\left(-2 S_{j}^{z}\right)
$$

and

$$
b_{i}^{+} b_{i+1}=a_{i}^{+} \prod_{j=1}^{i-1}\left(1-2 a_{j}^{+} a_{j}\right) \prod_{k=1}^{i}\left(1-2 a_{k}^{+} a_{k}\right) a_{i+1}=a_{i}^{+} a_{i+1}, \quad b_{i+1}^{+} b_{i}=a_{i+1}^{+} a_{i} .
$$

\footnotetext{
${ }^{1}$ First the "fermionization" was done in [12] for transformation between the spin variables and noninteracting lattice fermions. Jordan-Wigner transformation for spin systems in one and two dimensions was considered in [13]. It yields an explicit solution for spin correlation functions [14-18].
} 
Thus, we have free spinless fermions, whose Hamiltonian reads

$$
H=\sum_{i=1}^{N}\left(\varepsilon_{0}-\mu\right) a_{i}^{+} a_{i}+t_{0} \sum_{i=1}^{N}\left(a_{i}^{+} a_{i+1}+a_{i+1}^{+} a_{i}\right)
$$

Let us consider an infinite chain $(N \rightarrow \infty)$ and make Fourier transformation of operators in $(2)$

$$
a_{i}=\frac{1}{\sqrt{N}} \sum_{q} \mathrm{e}^{\mathrm{i} q R_{i}} \alpha_{q}, \quad a_{i}^{+}=\frac{1}{\sqrt{N}} \sum_{q} \mathrm{e}^{-\mathrm{i} q R_{i}} \alpha_{q}^{+}
$$

then $H$ has the diagonal form

$$
H=\sum_{q} \lambda(q) \alpha_{q}^{+} \alpha_{q}, \quad \lambda(q)=\varepsilon_{0}-\mu+2 t_{0} \cos (q a) .
$$

Here $a$ is the lattice constant , $q=(2 \pi n) /(N a), n=1, \ldots, N$ (throughout this paper $a=1)$.

It is easy to consider the thermodynamics of such a system in this representation. The grand canonical potential is

$$
\Omega=-\frac{1}{\beta} \ln \operatorname{Spe}^{-\beta H}=-\frac{1}{\beta} \ln \prod_{q} \sum_{n_{q}=0,1} \mathrm{e}^{-\beta \lambda(q) n_{q}}=-\frac{1}{\beta} \sum_{q} \ln \left(1+\mathrm{e}^{-\beta \lambda(q)}\right) .
$$

Then the average occupation number

$$
\langle n\rangle=\frac{1}{\pi} \int_{0}^{\pi}\left\langle n_{q}\right\rangle \mathrm{d} q, \quad\left\langle n_{q}\right\rangle=\frac{1}{2}-\frac{1}{2} \tanh \frac{\beta \lambda(q)}{2}
$$

and $\left\langle S^{z}\right\rangle=\langle n\rangle-1 / 2$.

\section{The time-dependent correlation functions on Pauli operators}

Let us consider the correlation functions built on Pauli operators. In order to calculate them we can use the results known for the time-dependent spin correlation functions. The exact numerical method of calculating such functions is described in [14-18]. In our case the parameter $\varepsilon=\varepsilon_{0}-\mu$ which can be introduced here plays the role of external field in spin representation. We investigate the ion chains with sufficiently large number of sites $N=400,600,1000$ to analyse the limit of large $N$.

Let us write the Hamiltonian (2) as follows:

$$
H=\sum_{i, j} a_{i}^{+} A_{i j} a_{j}
$$

where $A_{i j}=\left(\varepsilon_{0}-\mu\right) \delta_{i j}+t_{0}\left(\delta_{j, i+1}+\delta_{j, i-1}\right)$. The Hamiltonian (5) can be reduced to the diagonal form by a linear transformation

$$
a_{i}^{+}=\sum_{k} g_{i k} \eta_{k}^{+}, \quad a_{i}=\sum_{k} g_{i k} \eta_{k}, \quad H=\sum_{i, j, k, k^{\prime}} g_{i k} g_{j k^{\prime}} A_{i j} \eta_{k}^{+} \eta_{k^{\prime}},
$$

where $\eta_{k}^{+}, \eta_{k}$ are also Fermi operators.

Then a traditional eigenvalue problem takes place

$$
\sum_{i} g_{s i} A_{i l}=E_{s} g_{s l}
$$

which is solved using the standard numerical procedure. The Hamiltonian in diagonal form in this case is $H=\sum_{k=1}^{N} E_{k} \eta_{k}^{+} \eta_{k}$. Our task is to calculate the time dependent Pauli pair correlation functions. The relation

$$
\left\langle b(t) b^{+}(0)\right\rangle_{i}=\left\langle S^{-}(t) S^{+}(0)\right\rangle_{i}
$$


between Pauli and spin correlation functions is used. In this case

$$
\left\langle b(t) b^{+}(0)\right\rangle_{i}=2\left[\left\langle S^{x}(t) S^{x}(0)\right\rangle_{i}+\mathrm{i}\left\langle S^{x}(t) S^{y}(0)\right\rangle_{i}\right]
$$

and $\left\langle S^{x}(t) S^{x}(0)\right\rangle_{i}=\left\langle S^{y}(t) S^{y}(0)\right\rangle_{i},\left\langle S^{x}(t) S^{y}(0)\right\rangle_{i}=-\left\langle S^{y}(t) S^{x}(0)\right\rangle_{i}$.

Therefore, the calculation of the pair correlation functions on the Pauli operators is reduced to the calculation of the spin correlation functions of appropriate components. However, the computing of the spin correlation function maps into the calculation of the many-particle correlation function on Fermi operators.

Let us write down the fermionization of the $S_{i}^{x}, S_{i}^{y}$ operators:

$$
\begin{aligned}
& S_{i}^{x}=\frac{1}{2}\left(b_{i}^{+}+b_{i}\right)=\frac{1}{2} \prod_{j=1}^{i-1}\left(-2 S_{j}^{z}\right)\left(a_{i}^{+}+a_{i}\right), \\
& S_{i}^{y}=\frac{1}{2 i}\left(b_{i}^{+}-b_{i}\right)=\frac{1}{2 \mathrm{i}} \prod_{j=1}^{i-1}\left(-2 S_{j}^{z}\right)\left(a_{i}^{+}-a_{i}\right), \\
& S_{i}^{z}=\frac{1}{2}\left[b_{i}^{+}, b_{i}\right]=-\frac{1}{2}\left(b_{i}^{+}+b_{i}\right)\left(b_{i}^{+}-b_{i}\right)=-\frac{1}{2}\left(a_{i}^{+}+a_{i}\right)\left(a_{i}^{+}-a_{i}\right) .
\end{aligned}
$$

It is known that by substituting the expression for $S_{i}^{z}$ into $S_{i}^{x}$ and $S_{i}^{y}$ we can obtain a relation between spin correlation functions and the ones on the Fermi operators:

$$
\begin{aligned}
\left\langle S^{x}(t) S^{x}(0)\right\rangle_{i} & =\frac{1}{4}\left\langle\prod_{j=1}^{i-1} \varphi_{j}^{+}(t) \varphi_{j}^{-}(t) \varphi_{i}^{+}(t) \prod_{j=1}^{i-1} \varphi_{j}^{+} \varphi_{j}^{-} \varphi_{i}^{+}\right\rangle, \\
\left\langle S^{x}(t) S^{y}(0)\right\rangle_{i} & =\frac{i}{4}\left\langle\prod_{j=1}^{i-1} \varphi_{j}^{+}(t) \varphi_{j}^{-}(t) \varphi_{i}^{+}(t) \prod_{j=1}^{i-1} \varphi_{j}^{+} \varphi_{j}^{-} \varphi_{i}^{-}\right\rangle,
\end{aligned}
$$

where $\varphi_{j}^{ \pm}=a_{j}^{+} \pm a_{j}$. Wick's theorem can be applied to expand $\left\langle S^{x}(t) S^{x}(0)\right\rangle_{i}$ and $\left\langle S^{x}(t) S^{y}(0)\right\rangle_{i}$ into the sum of the products of elementary average values:

$$
\begin{aligned}
\left\langle\varphi_{i}^{+}(t) \varphi_{j}^{ \pm}\right\rangle & =\sum_{k=1}^{N} g_{i k} g_{j k}\left[\frac{\mathrm{e}^{\mathrm{i} E_{k} t}}{1+\mathrm{e}^{\beta E_{k}}} \pm \frac{\mathrm{e}^{-\mathrm{i} E_{k} t}}{1+\mathrm{e}^{-\beta E_{k}}}\right], \\
\left\langle\varphi_{i}^{+}(t) \varphi_{j}^{+}\right\rangle & =-\left\langle\varphi_{i}^{-}(t) \varphi_{j}^{-}\right\rangle, \quad\left\langle\varphi_{i}^{+}(t) \varphi_{j}^{-}\right\rangle=-\left\langle\varphi_{i}^{-}(t) \varphi_{j}^{+}\right\rangle .
\end{aligned}
$$

The expansions (9) and (10) can be more compactly expressed as a pfaffian [14-16].

The analytical results for these one-site spin correlation functions $\left\langle S^{x}(t) S^{x}(0)\right\rangle_{i},\left\langle S^{x}(t) S^{y}(0)\right\rangle_{i}$ were obtained by Capel and Perk [19] at the infinite temperature $\beta=0$ in the limiting case $N \rightarrow \infty$

$$
\left\langle S^{x}(t) S^{x}(0)\right\rangle=\frac{1}{4} \mathrm{e}^{-t_{0}^{2} t^{2}} \cos \left(\left(\varepsilon_{0}-\mu\right) t\right), \quad\left\langle S^{x}(t) S^{y}(0)\right\rangle=\frac{1}{4} \mathrm{e}^{-t_{0}^{2} t^{2}} \sin \left(\left(\varepsilon_{0}-\mu\right) t\right) .
$$

Substituting these relations into (8) we have

$$
\left\langle b(t) b^{+}(0)\right\rangle=\frac{1}{2} \mathrm{e}^{\varepsilon t} \mathrm{e}^{-t_{0}^{2} t^{2}} .
$$

For finite values of $N$, we obtain the correlation functions using a numerical method for calculation of pfaffians [15]. The transfer parameter $t_{0}$ is set equal to unity in this paper, $t_{0}=1$; all energy quantities are given in relation to $t_{0}$. Here we do not investigate the dependence of correlation on a site number. We consider only the one-site correlation at a separate site $(j=50$, taken as an example) and the boundary effects are neglected.

The time dependent correlation function $\left\langle b(t) b^{+}(0)\right\rangle_{j}$ at $j$ th site for the ion chain with $N=600$ and with the energy values $\varepsilon=\varepsilon_{0}-\mu=0.0001, \pm 0.5$ at $\beta=0.001$ is presented in figure 1 . The real 


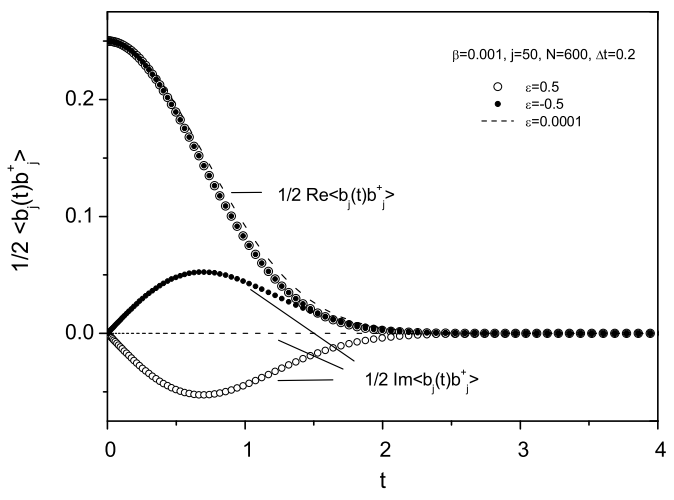

Figure 1. Time dependent correlation function $\left\langle b(t) b^{+}\right\rangle_{j}$ at $\beta=0.001$.

parts of Pauli correlation function are the same at $\varepsilon= \pm 0.5$ and the imaginary parts of $\left\langle b(t) b^{+}\right\rangle_{j}$ change the sign. $\operatorname{Im}\left\langle b(t) b^{+}(0)\right\rangle_{j}$ is equal practically to zero at $\varepsilon=0.0001$ and the correlation function is given by its real part.

The time dependence of the one-site Pauli correlation function becomes more complicated at finite temperatures. The case $\beta=1$ is presented in figure $2 ; \operatorname{Im}\left\langle b(t) b^{+}(0)\right\rangle_{j}$ is nonzero for $\varepsilon=0$. The oscillation appears at the increase of $\varepsilon$; the main minimum value of the imaginary part of the Pauli correlation function decreases with the energy $\varepsilon_{0}-\mu$ increase (figure $2 b$ ).
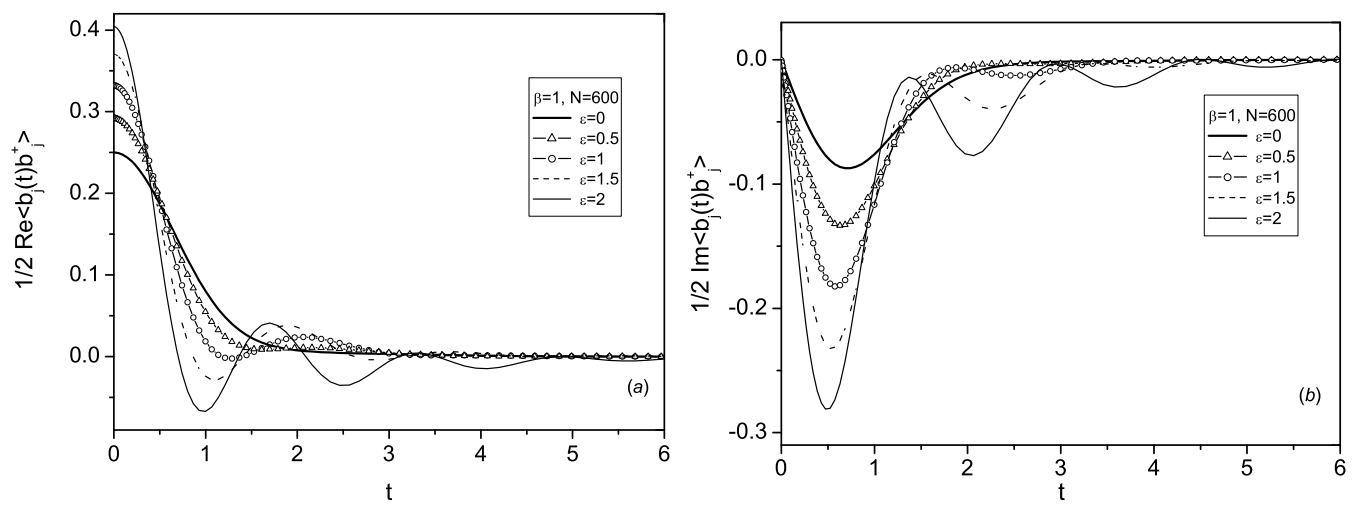

Figure 2. Time dependent correlation function $\left\langle b(t) b^{+}\right\rangle_{j}$ at $\beta=1$. (a): $\operatorname{Re}\left\langle b(t) b^{+}(0)\right\rangle_{j}$; (b): $\operatorname{Im}\left\langle b(t) b^{+}(0)\right\rangle_{j}$.

\section{Particle density of states}

The commutation relations for Pauli operators are intermediate between Fermi and Bose statistics. It is interesting to calculate the particle density of states (DOS) and compare with the mentioned pure cases. So, here we consider such a particle DOS as $\rho_{j}^{c}\left(\rho_{j}^{a}\right)$ calculated similarly to the case of bosons (fermions).

It is well known that the particle DOS can be obtained from the imaginary part of the two-time Zubarev Green's function:

$$
\rho_{i j}(\omega)=-2 \operatorname{Im}\left\langle\left\langle b_{i} \mid b_{j}^{+}\right\rangle\right\rangle_{\omega+\mathrm{i} \delta} .
$$

At first we consider the commutator Green's function

$$
G_{i j}\left(t, t^{\prime}\right)^{c}=\left\langle\left\langle b_{i}(t) \mid b_{j}^{+}\left(t^{\prime}\right)\right\rangle\right\rangle=-\mathrm{i} \Theta\left(t-t^{\prime}\right)\left\langle\left[b_{i}(t), b_{j}^{+}\left(t^{\prime}\right)\right]\right\rangle .
$$


The Fourier transforms of the Green's function in the spectral representation:

$$
\left\langle\left\langle b_{i} \mid b_{j}^{+}\right\rangle\right\rangle_{\omega+\mathrm{i} \delta}=\frac{1}{2 \pi} \int_{-\infty}^{+\infty} J_{b b^{+}}\left(\omega^{\prime}\right) \frac{1-e^{-\beta \omega^{\prime}}}{\omega-\omega^{\prime}+\mathrm{i} \delta} \mathrm{d} \omega^{\prime}
$$

is connected with the one-site autocorrelation function

$$
J_{b b^{+}}(\omega)=\int_{-\infty}^{+\infty} \mathrm{e}^{\mathrm{i} \omega\left(t-t^{\prime}\right)}\left\langle b(t) b^{+}\left(t^{\prime}\right)\right\rangle_{j} \mathrm{~d}\left(t-t^{\prime}\right) .
$$

The latter can be expressed in terms of spin operators

$$
J_{b b^{+}}(\omega)=\int_{-\infty}^{+\infty} \mathrm{e}^{\mathrm{i} \omega t}\left\langle S^{-}(t) S^{+}(0)\right\rangle_{j} \mathrm{~d} t
$$

Taking into account that

$$
\left\langle S^{-}(t) S^{+}(0)\right\rangle_{j}=\left\langle S^{-}(0) S^{+}(-t)\right\rangle_{j}=\left\langle S^{-}(0) S^{+}(t)\right\rangle_{j}^{*}=\left\langle S^{-}(-t) S^{+}(0)\right\rangle_{j}=\left\langle S^{-}(t) S^{+}(0)\right\rangle_{j}^{*},
$$

we have in Pauli operators

$$
J_{b b^{+}}(\omega)=\int_{0}^{\infty}\left[\mathrm{e}^{\mathrm{i} \omega t}\left\langle b(t) b^{+}(0)\right\rangle_{j}+\mathrm{e}^{-\mathrm{i} \omega t}\left\langle b(-t) b^{+}(0)\right\rangle_{j}\right] \mathrm{d} t=2 \operatorname{Re} \int_{0}^{\infty} \mathrm{e}^{\mathrm{i} \omega t}\left\langle b(t) b^{+}(0)\right\rangle_{j} \mathrm{~d} t .
$$

This expression is used for the numerical calculation of the particle autocorrelation function based on the procedure presented in the previous section.

In the analytical $(N \rightarrow \infty)$ case $(11)$ the autocorrelation function calculated using the expression (11) is as follows:

$$
J_{b b^{+}}(\omega)=\frac{\sqrt{\pi}}{2 t_{0}} \exp \left(\frac{(\omega-\varepsilon)^{2}}{4 t_{0}^{2}}\right)
$$

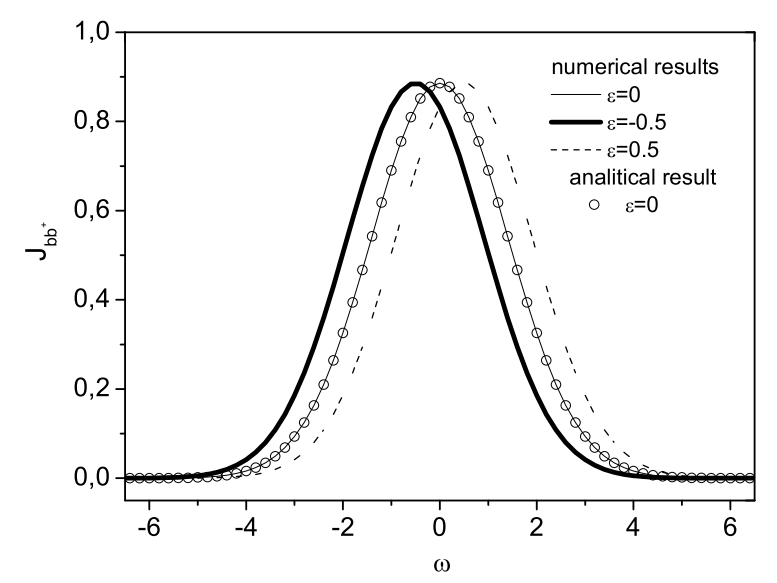

Figure 3. Frequency dependence of $J_{b b^{+}}(\omega)$ at $\beta=0.001$.

The frequency dependence of the $J_{b b^{+}}(\omega)$ at $\beta=0.001 ; \varepsilon=0.0001, \pm 0.5$ is presented in figure 3 . Here we compare the results of numerical calculations with the analytical ones (17) obtained at $\beta=0$. As it is shown in figure 3 on the example of the $\varepsilon=0$ case, numerical curves and analytical data are identical. The autocorrelation function $J_{b b^{+}}(\omega)$ possesses a gaussian shape with maximum value at $\omega=\varepsilon$; its positions shift to positive or negative values depending on the sign of the $\varepsilon$ parameter. 
Substitution of (14) into (12) gives the following expression for DOS

$$
\rho_{j}^{c}(\omega)=\left(1-\mathrm{e}^{-\beta \omega}\right) J_{b b^{+}}(\omega) .
$$

So, the calculation of the frequency dependent DOS $\rho_{j}^{c}$ is reduced to the calculation of the autocorrelation function (18) with a factor $1-\mathrm{e}^{-\beta \omega}$.
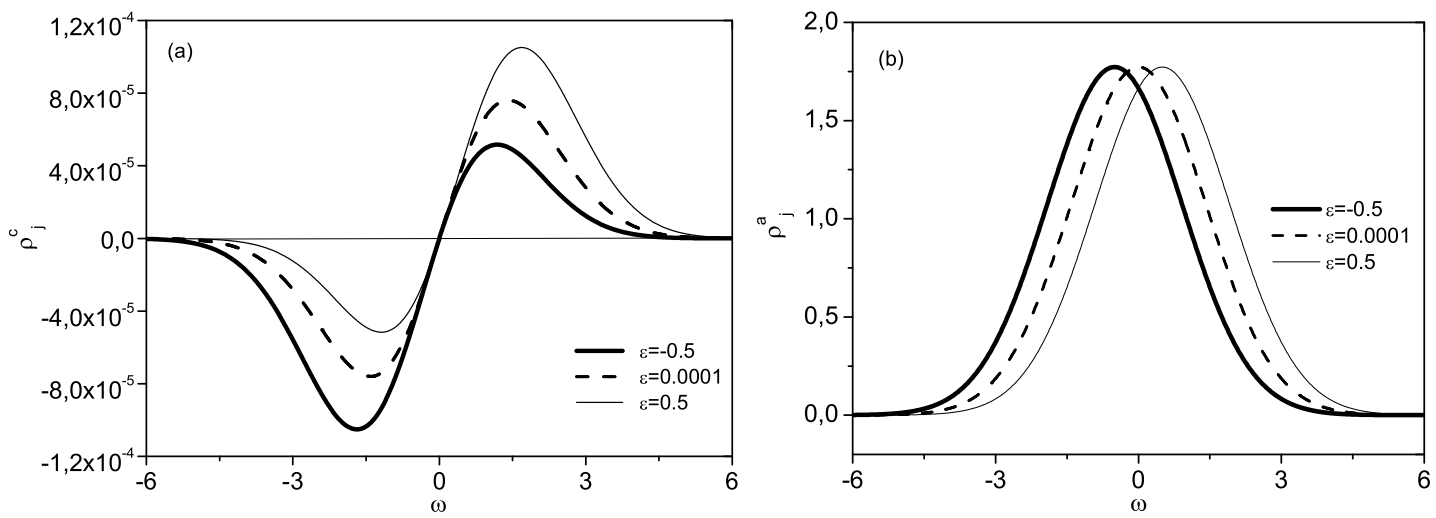

Figure 4. Frequency dependent $\operatorname{DOS} \rho_{j}^{c}, \rho_{j}^{a}$ at $\beta=0.001$.

The form of function $\rho_{j}^{c}(\omega)$ in the case $\beta=0.001$ is shown in figure 4 at $\varepsilon=0.0001, \pm 0.5$. The function changes its sign at $\omega=0$ and reaches maximum and minimum values at positive and negative frequencies, respectively. At $\varepsilon>0$ the spectral weight is larger at $\omega>0$ and increases at the increase of $\varepsilon$. For comparison in the same figure we show a plot of the "fermi-like" density of states

$$
\rho_{j}^{a}(\omega)=\left(1+\mathrm{e}^{-\beta \omega}\right) J_{b b^{+}}(\omega)
$$

as a function of frequency. The function $\rho_{j}^{a}(\omega)$ is connected with the anticommutator Green's function

$$
G_{i j}^{a}(\omega+\mathrm{i} \varepsilon)=-\mathrm{i} \theta\left(t-t^{\prime}\right)\left\langle\left\{b_{i}(t), b_{j}^{+}\left(t^{\prime}\right)\right\}\right\rangle
$$

by the relation similar to the relation (13). Its frequency behaviour is different from the $\rho_{j}^{c}(\omega)$ function coinciding up to a factor of 2 with the $J_{b b^{+}}$plot in the $\beta=0$ limit.

In figure 5 the frequency dependence of the autocorrelation function $J_{b b^{+}}(\omega)$ and density of states $\rho_{j}^{c}(\omega)$ is given in the case of finite temperatures $(\beta=1$ and $\beta=5)$ for different values of $\varepsilon$. At the temperature decrease (that corresponds to the increase of the $\beta$ parameter) the additional peaks appear on the frequency dependence of DOS (in the region of positive/negative frequencies at $\varepsilon>0 / \varepsilon<0)$. Their intensities grow at larger values of $|\varepsilon|$. This situation is also illustrated in figure 6 where a series of plots of the $J_{b b^{+}}(\omega)$ function at $\varepsilon=0.5$ at different temperatures is presented. At temperature lowering, two distinct peaks are developed. The similar two-peak structure also takes place at $\beta \gg 1$ for "fermi" DOS $\rho_{j}^{a}$. As an example, the plots of this function at $\varepsilon=-2$ are shown in figure 7 at different values of $\beta$.

The reason for such a behaviour of DOS at low temperatures is as follows. When $T \rightarrow 0$ and $\varepsilon \gg 1$ (we consider here, for definition, the case $\varepsilon>0$ ), the $b_{i}^{+}$and $b_{i}$ operators describe the creation and annihilation of the magnon-like excitations (at $\varepsilon>0$ the ground state of the system is realized at $S_{i}^{z}=-1 / 2$ ); their commutation relations become boson-like at low concentrations of "magnons". In this case the Hamiltonian (1) can be diagonalized at $N \rightarrow \infty$ in terms of $b_{i}^{+}, b_{i}$ operators by means of Fourier transformation with the eigenvalue spectrum, which coincides with formula (3). The density of states $\rho_{j}^{c}(\omega)$ is given now by the known expression

$$
\rho_{j}^{c}(\omega)=\frac{1}{N} \sum_{q} \delta(\omega-\lambda(q))=\frac{1}{\pi} \frac{1}{\sqrt{4 t_{0}^{2}-(\omega-\varepsilon)^{2}}}
$$



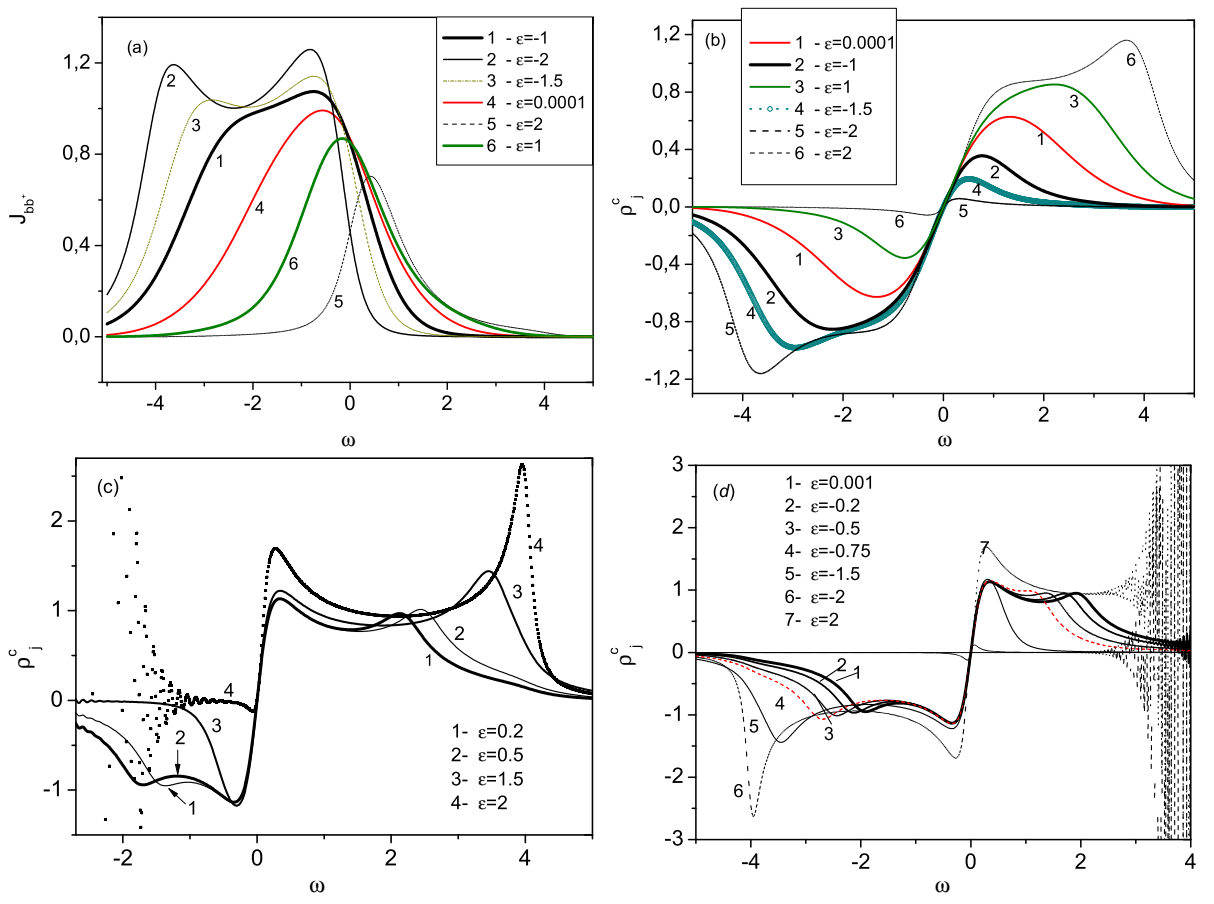

Figure 5. $J_{b b^{+}}(\omega), \rho_{j}^{c}(\omega)$ at $\beta=1(a, b), \rho_{j}^{c}(\omega)$ at $\beta=5(c, d)$.

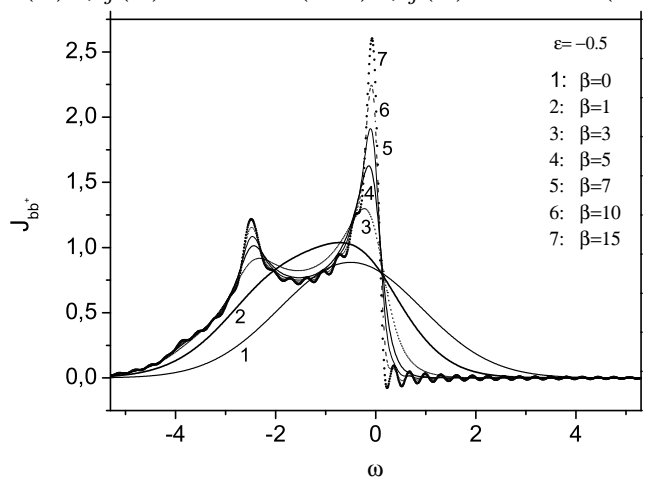

Figure 6. Autocorrelation functions $J_{b b^{+}}(\omega)$ at $\varepsilon=0.5$ for indicated temperatures.
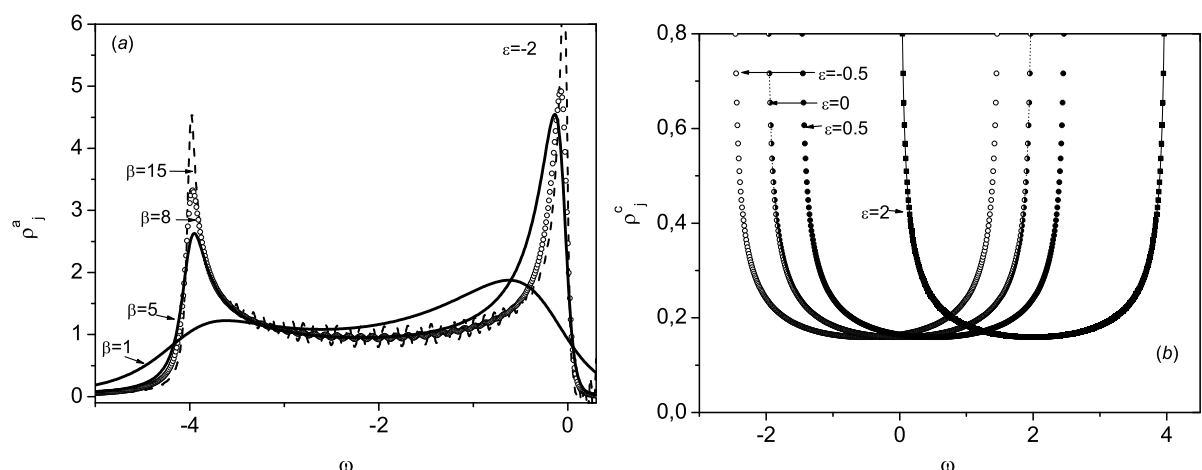

Figure 7. Frequency dependences $\rho_{j}^{a}(\omega)$ in the case $\varepsilon=-2$ at different temperatures (a) and $\left.\rho_{j}^{c}(\omega)\right|_{\beta \rightarrow \infty}$ at different values of $\varepsilon(\mathrm{b})$. 
at $|\omega-\varepsilon| \leqslant 2 t_{0}$. The function (21) diverges at the edges of the "magnon" band ( $\omega=\varepsilon \pm 2 t_{0}$, or $\omega-\varepsilon= \pm 1$ in dimensionless units). This result indicates the limiting shape of the two-peak structure of the $\rho_{j}^{c}(\omega)$ function when temperature goes to zero in the case of large values of $\varepsilon$.

Similarly we can interpret the form of the $\rho_{j}^{a}$ function at low temperatures. However, this function differs, on the whole, by its appearance from the $\rho_{j}^{c}$ one. Moreover, if the $b_{i}^{+}, b_{i}$ operators were considered as fermi-operators from the very beginning (which corresponds to the frequently used fermion lattice model), the eigenvalue spectrum would be the same as (3) and the fermion density of states $\rho_{j}^{a}(\omega)$ would be given by formula analogous to (21). Contrary to the case of Pauli operators, this should be the case in the whole temperature region.

\section{Conclusions}

The simple noninteracting model of ionic hopping in the one-dimensional chain is analysed in the given paper based on the lattice gas model. The particles are described using the Pauli statistics. The correlation functions constructed on the Pauli operators $b_{i}^{+}, b_{i}(0)$ of creation and annihilation of particles are investigated. The one-particle densities of states are calculated. An approach for correlation function $\left\langle b(t) b^{+}(0)\right\rangle_{j}$ is based on fermionization procedure which permits to formulate the exact calculation procedure. Numerical results for $\left\langle b(t) b^{+}(0)\right\rangle_{j}$ at $\beta=0$ are in good agreement with analytical estimates at $N \rightarrow \infty$. The frequency dependences of autocorrelation function $J_{b b^{+}}(\omega)$ as well as frequency dependences of densities of states are obtained for a fixed lattice site, temperature and particle energy. Special attention is paid to the changes in density of states at the decrease of temperature. The transformation of the one-particle spectrum (from gaussian-like density of states at high enough temperatures to the two-peak structure at low temperatures and sufficiently large energy difference $\varepsilon=\varepsilon_{0}-\mu$ ) is analysed. The correlation between the two-peak DOS and density of states $\rho_{j}^{c}$ at $T \rightarrow 0,|\varepsilon| \rightarrow \infty$, that corresponds to the boson gas of the magnonlike excitations, is established. A comparison with the DOS in the fermion picture shows significant differences in spectrum and its spectral features.

\section{Acknowledgements}

The authors are greatly indebted to Taras Krokhmalskii for help, collaboration and numerical assistance. 


\title{
References
}

1. Mahan G. D., Phys. Rev. B, 1976, 14, No. 2, 780.

2. Yonashiro K., Tomoyose T., Sakai E., J. Phys. Soc. Jpn., 1983, 52, No. 11, 3837.

3. Tomoyose T., J. Phys. Soc. Jpn., 1990, 60, No. 4, 1263.

4. Tomoyose T., J. Phys. Soc. Jpn., 1997, 66, No. 8, 2383.

5. Tanaka T., Majed A. Sawatarie, Barry J. H., Shatma N. L. and Munera C. H., Phys. Rev. B, 1986, 34, 3773 .

6. I. V. Stasyuk, O. L. Ivankiv, N. I. Pavlenko, J. Phys. Stud., 1997, 1, 418.

7. Yu. I. Dublenych, Phys. Rev. B., 2005, 71, 012411.

8. Rikvold P. A., Zhang J., Sung Y. E., Wieckowski A. Preprint arXiv:cond-mat/9506025, 1995.

9. I. V. Stasyuk, N. I. Pavlenko, J. Chem. Phys., 2001, 114, No. 10, 4607.

10. R. Micnas and S. Robaszkiewicz,Phys. Rev. B., 1992, 45, No. 17, 9902.

11. I. V. Stasyuk, O. Vorobyov, B. Hilczer, Solid State Ionics, 2001, 145, 211.

12. Lieb E., Schultz T., Mattis D., Ann. Phys., 1961, 16, 407.

13. Derzhko O., J. Phys. Stud., 2001, 5, No. 1, 49.

14. Derzhko O., Krokhmalskii T., Phys. Stat. Sol. B, 1998, 208, 221.

15. Derzhko O., Krokhmalskii T., Phys. Stat. Sol. B, 2000, 217, 927.

16. Derzhko O., Krokhmalskii T., J. Phys. A, 2000, 33, 3063.

17. Stolze J., Vogel M., Phys. Rev. B, 2000, 61, 4026.

18. Derzhko O., Krokhmalskii T. and Stolze J., J. Phys. A, 2002, 35, 3573.

19. Capel H. W., Perk J. H. H., Physica A, 1977, 87, 211.

\section{Густина станів одновимірного іонного провідника Паулі}

\author{
І.В.Стасюк, І.Р.Дулепа \\ Інститут фізики конденсованих систем НАН України, 79011 Львів, вул. І.Свєнціцького, 1 \\ Отримано 7 травня 2007 р.
}

Розглянуто мікроскопічну одновимірну модель невзаємодіючих частинок для опису енергетичного спектру іонної підсистеми в іонному провіднику. Процеси іонного перескоку описані у термінах операторів Паулі. Використовуючи точний числовий метод, відомий для часових спінових кореляційних функцій, отримано часові кореляційні функції $\left\langle b(t) b^{+}(0)\right\rangle_{j}$ в операторах Паулі. Обчислено і проаналізовано частотну залежність автокореляційної функції $J_{b b}+(\omega)$ в широкому інтервалі температур. Досліджено частотну і температурну залежності одночастинкової густини станів.

Ключові слова: статистика Паулі, кореляційні функції, частинкова густина станів, іонний провідник

PACS: $75.10 . P q, 66.30 . D n, 66.10 . E d$ 